\title{
Pulmonary mucormycosis: A case report and review of the literature
}

\author{
XI-MING WANG ${ }^{1}$, LING-CHUAN GUO ${ }^{2}$, SHENG-LI XUE $^{3}$ and YAN-BIN CHEN ${ }^{4}$ \\ Departments of ${ }^{1}$ Radiology, ${ }^{2}$ Pathology, ${ }^{3}$ Hematology and ${ }^{4}$ Respiratory Medicine, \\ The First Affiliated Hospital of Soochow University, Suzhou, Jiangsu 215006, P.R. China
}

Received February 1, 2015; Accepted February 19, 2016

DOI: $10.3892 / \mathrm{ol} .2016 .4370$

\begin{abstract}
The current study reports the case of a 15-year-old male who presented to The First Affiliated Hospital of Soochow University (Suzhou, Jiangsu, China) with a 3-day history of anergy and epistaxis. The patient was diagnosed with T-cell acute lymphoblastic leukemia according to the results of a bone marrow examination and received chemotherapy. During the agranulocytosis period, the patient developed pneumonia of the right upper lung (RUL). Once complete remission was achieved, the patient underwent a lobectomy of the RUL, together with amphotericin B therapy, following the confirmation of pulmonary mucormycosis by the histopathological results. The patient experienced 12 months of uneventful follow-up post-surgery.
\end{abstract}

\section{Introduction}

Mucormycosis is an opportunistic infection that is caused by Mucorales fungi of the Zygomycetes class. The term zygomycosis, the previous designation for infections caused by fungi of the order Mucorales, is no longer appropriate due to a recent taxonomic reclassification that abolished Zygomycetes as a class (1). Mucorales fungi are ubiquitous, saprophytic and not fastidious fungi located in soil or decaying organic matter, with three genera that are known to be human pathogens, namely, Rhizopus, Absidia and Mucor. The optimal temperature for growth is 28 to $30^{\circ} \mathrm{C}$ under aerobic conditions, with an incubation period of 2 to 5 days. Incubation begins with inhalation of the spores or their direct inoculation into abraded skin (2). Six distinct clinical presentations of mucormycosis are now recognized: Rhinocerebral, cutaneous, pulmonary, gastrointestinal and central nervous system mucormycosis, and a miscellaneous form involving the bones, breasts, mediastinum

Correspondence to: Mr. Yan-Bin Chen, Department of Respiratory Medicine, The First Affiliated Hospital of Soochow University, 188 Shizi Street, Suzhou, Jiangsu 215006, P.R. China

E-mail: chen001chen@163.com

Key words: pulmonary mucormycosis, lobectomy, amphotericin B, air-crescent sign and kidneys. The first case of pulmonary mucormycosis was described in 1876 by Furbringer (3). The estimated incidence of the disease is 1.7 cases per million people per year in the United States (4). In a review of 116 cases of mucormycosis, $22 \%$ were pulmonary mucormycosis (5). However, the incidence of pulmonary mucormycosis has increased with the development of modern medicine. Numerous predisposing clinical factors have been described, including uncontrolled diabetes mellitus, diabetic ketoacidosis, chemotherapy, hematological malignancies (leukemia and lymphoma), immunosuppressive therapy, acquired or congenital neutropenia, antibiotic therapy, metabolic acidosis due to chronic salicylate poisoning, elastoplast bandages, renal failure, a prolonged post-operative course, solid tumors, solid organ transplantation, agammaglobulinemia and burns (6-8). It is well known that iron metabolism has a key role in mucormycosis pathogenesis. Therefore, patients in an iron overload state, including those individuals undergoing deferoxamine chelation therapy, are uniquely predisposed to mucormycosis (9). Only $6.25 \%$ patients do not have any underlying risk factor $(10,11)$. In patients with hematological malignancies, mucormycosis most commonly affects the lungs (58-81\%) (12). A previous single-center autopsy study over a 15 -year period in patients with hematological malignancy reported a significant 3-fold increase in the incidence of autopsy-proven mucormycosis cases, from $0.9-3 \%$, during the study period (13). The present study reports the case of a patient with a definite histological diagnosis of pulmonary mucormycosis.

\section{Case report}

A previously healthy 15-year-old male was admitted to The First Affiliated Hospital of Soochow University (Suzhou, Jiangsu, China) in January 2012 with a 3-day history of anergy and epistaxis. There was no history of hemoptysis, fever, chills, night sweats, chest pain, diabetes mellitus or weight loss. The patient reported no history of steroid use. Physical examination showed a well-developed male with facial pallor and fresh petechia on the lower limbs. The patient had a temperature of $36.7^{\circ} \mathrm{C}$ (normal range, $36-37^{\circ} \mathrm{C}$ ), a pulse rate of 80 beats $/ \mathrm{min}$ (normal range, $60-100$ beats/min), a respiratory rate of 20 breaths $/ \mathrm{min}$ (normal range, 12-20 breaths/min) and a blood pressure of $120 / 60 \mathrm{mmHg}$ (normal range, $<130 / 85 \mathrm{mmHg}$ ). Further physical examination results were unremarkable. The chest radiograph 


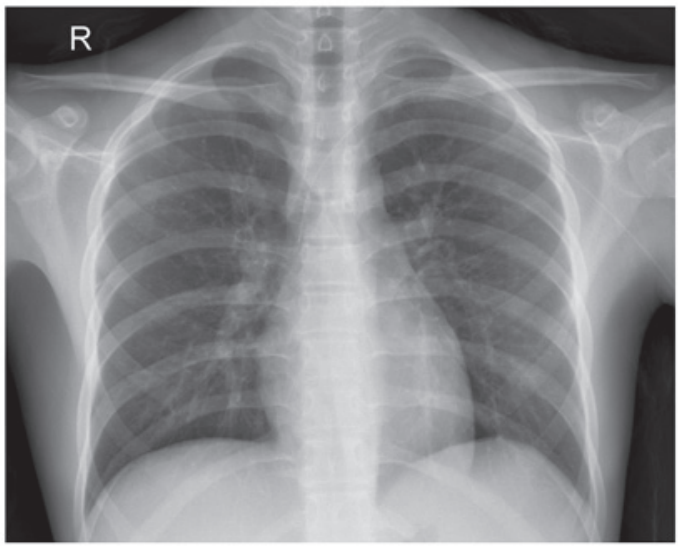

Figure 1. Chest radiograph at the time of hospital admission showing no infiltration in either side of the lung.

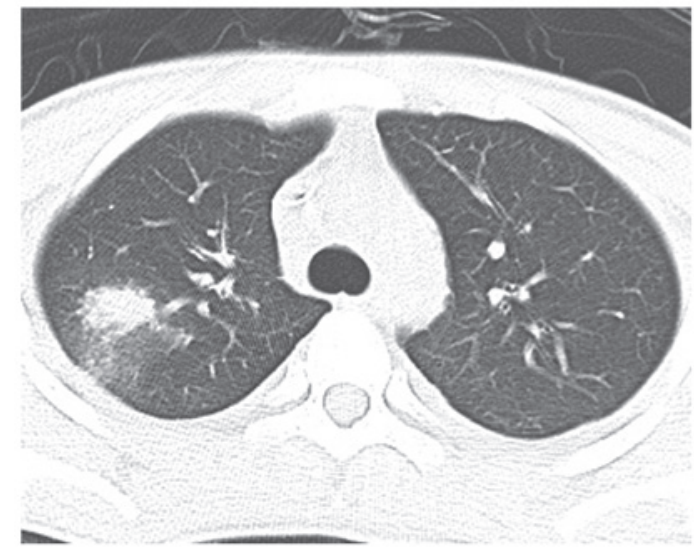

Figure 2. Chest computed tomography scan on day 15 post-admission showing a focal area of consolidation with surrounding ground-glass opacity (halo sign) in the posterior segment of the right upper lobe.

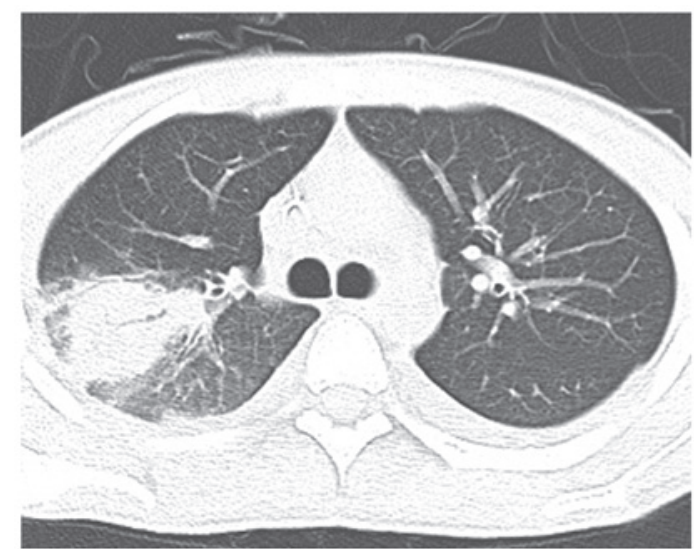

Figure 3. Chest computed tomography scan on day 23 post-admission showing an increase in size of the previously demonstrated consolidation, which has extended to the visceral pleura, resulting in pleural thickening.

showed no abnormalities (Fig. 1). A full blood count revealed a hemoglobin level of $76 \mathrm{~g} / 1$ (normal range, 120-150 g/l), a platelet count of $1.5 \times 10^{10} / 1$ (normal range, $10.0-30.0 \times 10^{10} / \mathrm{l}$ ) and a white blood cell count of $1.24 \times 10^{11} / 1$ (normal range, $4.00-10.00 \times 10^{9} / 1$ ), with $1.24 \times 10^{9} / 1$ neutrophils (normal range,

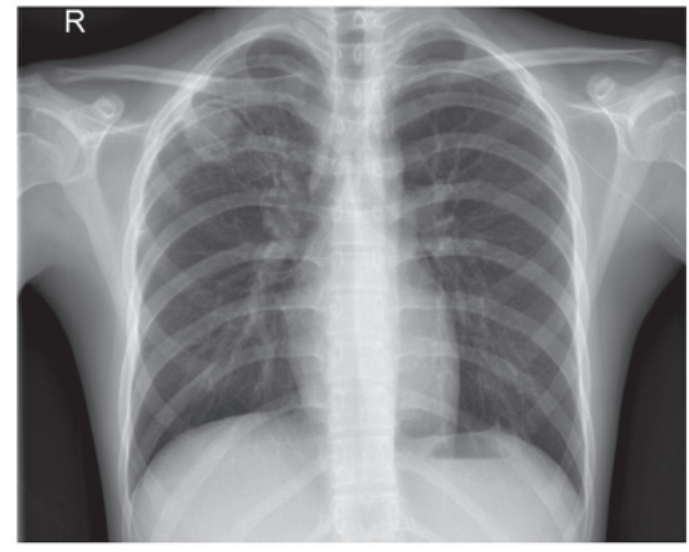

Figure 4. Follow-up chest radiograph obtained 2 weeks after Fig. 3 (day 37) showing a ball-shaped cavitating mass with crescentic cavitation in the posterior segment of the right upper lobe.

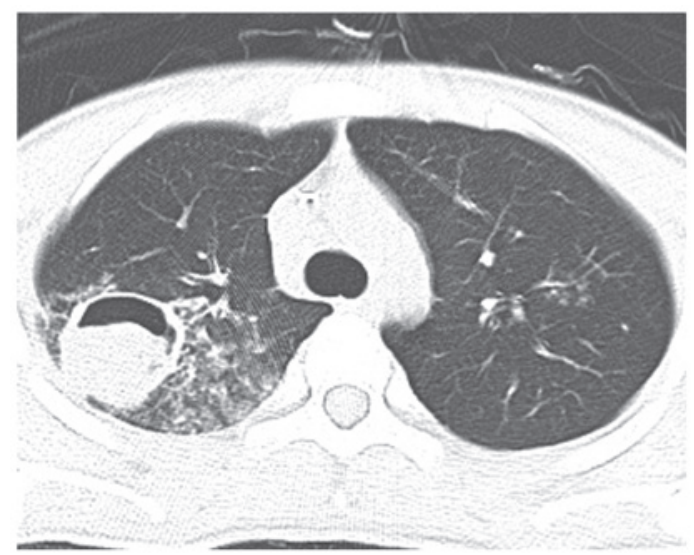

Figure 5. Chest computed tomography scan on the same day as Fig. 4 (day 37) showing a well-defined cavitating mass within the right upper lobe, with the air-crescent sign. Nodules of varying sizes are noted around the lesion and in the left upper lobe.

$\left.1.80-6.30 \times 10^{9} / 1\right), 2.98 \times 10^{10} / 1$ lymphocytes (normal range, 1.10 $3.20 \times 10^{10} / 1$ ), and $9.3 \times 10^{10} / 1$ protocells and juvenile cells (normal range, 0 cells). Bone marrow examination revealed $89.1 \%$ of the naive population were $\mathrm{T}$ lymphocytic cells (normal range, $0 \%$ ). The patient was therefore diagnosed with T-cell acute lymphoblastic leukemia (T-ALL). At 3 days post-evaluation, the patient received induction chemotherapy with an IVP regimen (10 mg idamycin on days 1-4; $4 \mathrm{mg}$ vindesine once a week for four weeks; and $10 \mathrm{mg}$ dexamethasone every day). On the 15th hospital day the patient developed a fever, with a temperature of $39^{\circ} \mathrm{C}$. Computed tomography (CT) scans showed right upper lobe (RUL) infiltration (Fig. 2). The white blood cell count was $0.3 \times 10^{9} / 1$ and the neutrophil count was $0.03 \times 10^{9} / 1$. Throat swab, blood, urine and sputum cultures for bacteria and fungus were repeatedly obtained, but did not reveal any pathogens. Sputum smears for acid-fast bacilli were negative, and galactomannan testing (GM) for diagnosing invasive aspergillosis was negative. The patient clinically improved following intravenous meropenem $(0.5 \mathrm{~g}$; every $8 \mathrm{~h})$, teicoplanin $(3 \mathrm{mg} / \mathrm{kg}$; every $12 \mathrm{~h})$, amphotericin B (1 mg on day 1 , then add $5 \mathrm{mg}$ every day until $0.5 \mathrm{mg} / \mathrm{kg} /$ day reached) and caspofungin (70 $\mathrm{mg}$ on day 1 , then $50 \mathrm{mg}$ /day) empirically 


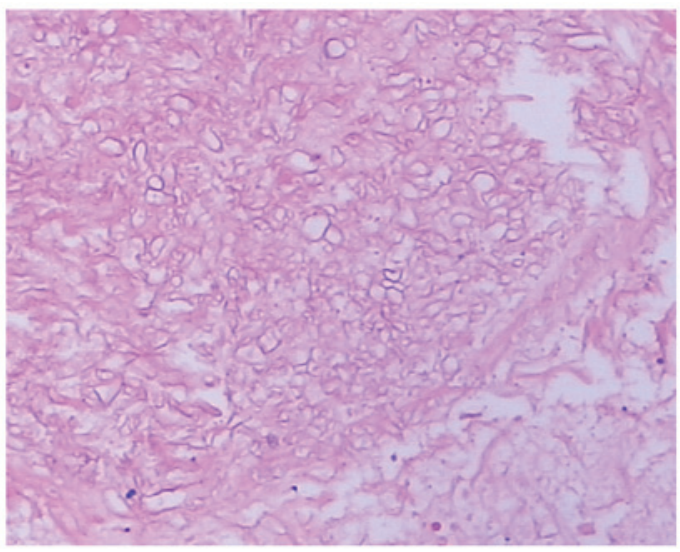

Figure 6. Lung resection specimen showing confluent fungal granulomata formed of colonies of broad, non-septate, right-angled branching hyphae, with oval conidia, within eosinophilic necrotic material consistent with mucormycosis (hematoxylin and eosin staining; magnification, $\mathrm{x} 40$ ).

for one week, however, radiography showed progression of the infiltrating lesion in the RUL (Figs. 3-5). Following chemotherapy with L-asparaginase (10,000 units every other day, three times), the patient had a white blood cell count of $8.27 \times 10^{9} / 1$, a hemoglobin level of $84 \mathrm{~g} / 1$ and a platelet count of $195 \times 10^{9} / 1$. Bone marrow morphology showed complete remission had been attained. The patient underwent a lobectomy of the RUL at 40 days post-admission, during which the infected area and necrotic tissue were resected. During surgery, a mass measuring $5 \times 5 \times 5 \mathrm{~cm}$ was revealed arising from the posterior segment of the RUL, which was tightly adherent to the chest wall. The pulmonary hilar lymph nodes were slightly enlarged. Histological study of the mass using hematoxylin and eosin staining revealed features consistent with pulmonary mucormycosis. The mass was composed of a large amount of right-angled branching, broad, non-septate hyphae. Epithelioid cells and an intense chronic inflammatory reaction were noted (Fig. 6). The patient experienced 12 months of uneventful follow-up post-surgery; however, the patient died from severe septic shock 13 months following surgery.

Written informed consent was obtained from the patient for the publication of the study.

\section{Discussion}

The present study reports the case of a young boy with T-ALL that developed agranulocytosis following chemotherapy at the same time as pneumonia. RUL infiltration progressed after empirical antibiotic therapy, which included antifungal agents. Surgery was the first choice of treatment for the patient and, fortunately, pulmonary mucormycosis was diagnosed subsequent to the lobectomy, so amphotericin B therapy was continuously provided. The patient's pulmonary mucormycosis was successfully treated.

Pulmonary mucormycosis occurs due to the inhalation of fungi spores into the bronchioles and alveoli, which typically results in the rapid progression of pneumonia or endobronchial disease. Rarer results include endobronchial lesions and complications associated with airway occlusion. Hemoptysis commonly occurs with vascular invasion, which can occasionally be fatal. The symptoms of pulmonary mucormycosis are typically non-specific, even at late stages of infection, and may include fever, dyspnea, coughing and chest pain. Rare cases can present as progressive subcutaneous emphysema, Pancoast syndrome, Horner's syndrome, or chronic mediastinitis and bronchial perforation (7,14-17).

The radiological manifestations of pulmonary mucormycosis are mostly non-specific. An abnormal chest roentgenogram result is present in $>80 \%$ of patients (18). The reported findings include consolidation, cavitation, the air-crescent sign, the halo sign, the reversed halo sign, solitary or multiple pulmonary nodules or masses, bronchopleural fistulae, pulmonary artery pseudoaneurysms, lymphadenopathy and pleural effusion. Cavitation is observed in as many as $40 \%$ of cases, but the air-crescent sign is uncommon. CT can show findings that alter the management or diagnostic approach in as many as $26 \%$ of patients (19-24). The presence of the air-crescent sign often portends a poor prognosis if surgical therapy is delayed. Similar to invasive pulmonary aspergillosis, pulmonary mucormycosis is detected with the highest sensitivity when using high-resolution chest CT to determine the extent of the disease. This technique also usually finds evidence of the infection earlier than standard chest radiographs $(2,4,9)$. The right lung is more commonly involved than the left, and there is a predilection for the involvement of the upper lobes, although the reason for this remains unknown. The present case reported a lesion in the RUL, as in the majority of the cases in the literature (25).

Histopathologically, vascular invasion with tissue necrosis and neutrophilic infiltration of the tissue is common to all types of mucormycosis. Diagnosis is achieved by demonstrating broad (diameter, 6-16 $\mu \mathrm{m}$ ), non-septate (coenocytic), ribbon-like hyphae, with right-angled branching in a tissue biopsy specimen stained with routine hematoxylin and eosin. Special fungal stains are usually not necessary for diagnosis. The less common and less specific features of pulmonary mucormycosis include bronchial invasion, pneumonia, lung abscesses and granulomatous pneumonitis $(20,21)$.

As pulmonary mucormycosis demonstrates rapid clinical progression and is often fatal, patient survival is dependent on an early diagnosis. Mucorales fungi are ubiquitous saprophytic fungi that grow in decaying organic matter, particularly fruit with a high sugar content, soil and manure. Although the fungi are able to grow in anaerobic, aerobic and microaerophilic conditions, clinical specimen cultures often prove to be negative, making the diagnosis difficult. There has previously been no serological test for mucormycosis. The symptoms, signs and radiographic manifestations of pulmonary mucormycosis are non-specific. Pulmonary mucormycosis is associated with bacterial pneumonia in $30 \%$ of cases, which can delay the diagnosis of the fungal infection (26). Diagnostic options are largely limited to the clinical and radiographic findings, together with staining and culture. A definitive diagnosis depends on the identification of mucoraceus hyphae in affected tissues (9,21); diagnostic techniques used to achieve this identification include percutaneous needle biopsy, open lung biopsy and pleural fluid culture. Fiberoptic bronchoscopy is a useful diagnostic method, and an adequate bronchoalveolar lavage specimen provides enough diagnostic material to form a cytological diagnosis (27). The differential diagnosis of the 
disease includes bacterial, viral and other fungal pulmonary infections. Pulmonary mucormycosis has clinical manifestations for which there is almost no differentiation from those of other more common opportunistic molds such as Aspergillus. The differentiation between mucormycosis and aspergillosis is important as the treatments can differ, and since the patient outcome may be improved by the appropriate early treatment of mucormycosis (19). In previous studies, polymerase chain reaction analysis from deparaffinized sections performed from a selected paraffin block showing both subtypes of hyphae allowed the identification of aspergillosis and mucormycosis $(28,29)$.

Successful treatment of pulmonary mucormycosis relies on a timely diagnosis. Amphotericin B, along with surgical resection of the involved areas of the lung and treatment of the underlying disease, is the mainstay of treatment (25). Despite the risk of renal toxicity, amphotericin B (1-1.5 mg/kg/day) remains the gold-standard antifungal agent used against mucormycosis. Oral posaconazole is also recommended, but these two types of drugs are often ineffective without surgical intervention (9,10,30-32). Voriconazole is ineffective against mucormycosis (33). Although the therapy duration is not well defined, a total cumulative dose of $1.5 \mathrm{~g}$ of amphotericin is usually sufficient in the selective group of patients who respond only to amphotericin therapy (34). Surgical therapy, such as wedge resection, lobectomy and pneumonectomy, in combination with medical therapy, has been associated with lower mortality rates in published series of patients with Mucor infection, particularly in patients with disease confined to one lung (35-38). In order to prevent dissemination and erosion into the vessels, which can result in potentially fatal massive hemoptysis, surgery should be performed as soon as possible (39).

Unlike pulmonary aspergillosis, pulmonary mucormycosis has a prognosis and outcome that have not significantly improved over the last decade, mainly due to the difficulty in forming an early diagnosis and the limited activity of current antifungal agents against Mucorales $(9,11)$. Pulmonary mucormycosis is a rapidly fatal illness, with an overall mortality rate of $76 \%$, which increases to $95 \%$ with extrathoracic dissemination (5,7,9,40-43). If untreated, survival beyond 2 weeks is distinctly unusual (40). In total, $<50 \%$ of patients are diagnosed premortem (18). Delays in the diagnosis result in a lethal clinical course due to fungal sepsis, respiratory failure and hemoptysis $(44,45)$. The outcome is typically fatal when pulmonary mucormycosis develops in a patient with hematological disease (41). Combined surgical/medical treatment may provide a better survival outcome than medical therapy alone (46).

\section{References}

1. Hibbett DS, Binder M, Bischoff JF, Blackwell M, Cannon PF Eriksson OE, Huhndorf S, James T, Kirk PM, Lücking R, et al: A higher-level phylogenetic classification of the Fungi. Mycol Res 111: 509-547, 2007.

2. Spellberg B, Edwards J Jr and Ibrahim A: Novel perspectives on mucormycosis: Pathophysiology, presentation and management. Clin Microbiol Rev 18: 556-569, 2005.

3. Fürbringer P: Observations on pulmonary mucormycosis in humans. Virchows Arch Path Anat 66: 330-365, 1876 (In German).

4. Garg R, Marak RS, Verma SK, Singh J, Sanjay and Prasad R: Pulmonary mucormycosis mimicking as pulmonary tuberculosis: A case report. Lung India 25: 129-131, 2008.
5. Aboutanos MB, Joshi M and Scalea TM: Isolated pulmonary mucormycosis in a patient with multiple injuries: A case presentation and review of the literature. J Trauma 54: 1016-1059, 2003.

6. Bigby TD, Serota ML, Tierney LM Jr and Matthay MA: Clinical spectrum of pulmonary mucormycosis. Chest 89: 435-439, 1986.

7. Muqeetadnan M, Rahman A, Amer S, Nusrat S, Hassan S and Hashmi S: Pulmonary mucormycosis: An emerging infection. Case Rep Pulmonol 2012: 120809, 2012.

8. Mohammadi A, Mehdizadeh A, Ghasemi-Rad M Habibpour H and Esmaeli A: Pulmonary mucormycosis in patients with diabetic ketoacidosis: A case report and review of literature. Tuberk Toraks 60: 66-69, 2012

9. Hamilos G, Samonis G and Kontoyiannis DP: Pulmonary mucormycosis. Semin Respir Crit Care Med 32: 693-702, 2011.

10. von Scheven R, Lebiedz P, Spieker T, Uekoetter A, Berdel WE and Kessler T: Fulminant invasive pulmonary mucormycosis with Rhizopus oryzae in a patient with severe aplastic anaemia and common variable immunodeficiency. Mycoses 55: e32-e35, 2012.

11. Roden MM, Zaoutis TE, Buchanan WL, Knudsen TA, Sarkisova TA, Schaufele RL, Sein M, Sein T, Chiou CC, Chu JH, et al: Epidemiology and outcome of zygomycosis: A review of 929 reported cases. Clin Infect Dis 41: 634-653, 2005.

12. Pagano L, Offidani M, Fianchi L, Nosari A, Candoni A, Piccardi M, Corvatta L, D'Antonio D, Girmenia C, Martino P, et al: Mucormycosis in hematologic patients. Haematologica 89: 207-214, 2004.

13. Chamilos G, Luna M, Lewis RE, Bodey GP, Chemaly R, Tarrand JJ, Safdar A, Raad II and Kontoyiannis DP: Nvasive fungal infections in patients with hematologic malignancies in a tertiary care cancer center: An autopsy study over a 15 -year period (1989-2003). Haematologica 91: 986-989, 2006.

14. Bansal M, Martin SR, Rudnicki SA, Hiatt KM and Mireles-Cabodevila E: A rapidly progressing Pancoast syndrome due to pulmonary mucormycosis: A case report. J Med Case Rep 5: 388, 2011.

15. Koshy CG, Shah S and Mammen T: Subcutaneous emphysema of the chest: Could it be pulmonary mucormycosis? Thorax 65: 280,2010

16. Kotoulas C, Psathakis K, Tsintiris K, Sampaziotis D, Karnesis L and Laoutidis G: Pulmonary mucormycosis presenting as Horner's syndrome. Asian Cardiovasc Thorac Ann 14: 86-87, 2006.

17. Liu HC, Jan MS, Lin YC, Lin WL, Wu TC, Huang CN, Chen CM and Lu MC: A rare pulmonary zygomycosis manifested as chronic mediastinitis and bronchial perforation. Eur Respir J 38: 734-735, 2011.

18. Donado-Uña JR, Díaz-Hellín V, López-Encuentra A and Echave-Sustaeta JM: Persistent cavitations in pulmonary mucormycosis after apparently successful amphotericin B. Eur J Cardiothorac Surg 21: 940-942, 2002.

19. Chung JH, Godwin JD, Chien JW and Pipavath SJ: Case 160: Pulmonary mucormycosis. Radiology 256: 667-670, 2010.

20. McAdams HP, Rosado de Christenson M, Strollo DC and Patz EF Jr: Pulmonary mucormycosis: Radiologic findings in 32 cases. AJR Am J Roentgenol 168: 1541-1548, 1997.

21. Walsh TJ, Gamaletsou MN, McGinnis MR, Hayden RT and Kontoyiannis DP: Early clinical and laboratory diagnosis of invasive pulmonary, extrapulmonary and disseminated mucormycosis (zygomycosis). Clin Infect Dis 54 (Suppl 1): S55-S60, 2012.

22. Ono A, Okada F, Ando Y, Maeda T, Saburi Y, Kondo Y and Mori H: Multiple pulmonary arteriolar emboli in a patient with disseminated mucormycosis and myelodysplastic syndrome. Clin Radiol 66: 998-1000, 2011.

23. Godoy MC and Marom EM: Reversed halo sign in pulmonary zygomycosis. Thorax 66: 544, 2011.

24. Busca A, Limerutti G, Locatelli F, Barbui A, De Rosa FG and Falda M: The reversed halo sign as the initial radiographic sign of pulmonary zygomycosis. Infection 40: 77-80, 2012.

25. Butala A, Shah B, Cho YT and Schmidt MF: Isolated pulmonary mucormycosis in an apparently normal host: A case report. J Natl Med Assoc 87: 572-574, 1995.

26. Pavie J, Lafaurie M, Lacroix C, Marie Zagdanski A, Debrosse D, Socié G, Derouin F, Gluckman E and Michel Molina J: Successful treatment of pulmonary mucormycosis in an allogenic bone-marrow transplant recipient with combined medical and surgical therapy. Scand J Infect Dis 36: 767-769, 2004.

27. al-Abbadi MA, Russo K and Wilkinson EJ: Pulmonary mucormycosis diagnosed by bronchoalveolar lavage: A case report and review of the literature. Pediatr Pulmonol 23: 222-225, 1997. 
28. Hofman V, Dhouibi A, Butori C, Padovani B, Gari-Toussaint M Garcia-Hermoso D, Baumann M, Vénissac N, Cathomas G and Hofman P: Usefulness of molecular biology performed with formaldehyde-fixed paraffin embedded tissue for the diagnosis of combined pulmonary invasive mucormycosis and aspergillosis in an immunocompromised patient. Diagn Pathol 5: 1, 2010.

29. Kobayashi M, Togitani K, Machida H, Uemura Y, Ohtsuki Y and Taguchi H: Molecular polymerase chain reaction diagnosis of pulmonary mucormycosis caused by Cunninghamella bertholletiae. Respirology 9: 397-401, 2004.

30. Uchida Y, Tsukino M, Shigemori W, Hayashi E, Watanabe I, Nakayama T, Yamada E and Moro K: Diagnosis of pulmonary mucormycosis aiding the diagnosis of small cell lung cancer. J Med Microbiol 61: 1610-1613, 2012.

31. Brugière O, Dauriat G, Mal H, Marrash-Chalha R, Fournier M, Groussard $\mathrm{O}$, Besnard $\mathrm{M}$, Lesèche $\mathrm{G}$ and Dupont B: Pulmonary mucormycosis (zygomycosis) in a lung transplant recipient: Recovery after posaconazole therapy. Transplantation 80 1361-1362, 2005

32. Sharma SK, Agarwal N, Mukherjee A, Seth T, Mishra P, Xess I, Mahapatra M and Sharma S: Coexisting pulmonary tuberculosis and mucormycosis in a patient with aplastic anemia post allogenic stem cell transplantation. Mediterr J Hematol Infect Dis 3: e2011036, 2011.

33. Fanci R, Pecile P, Di Lollo S, Dini C and Bosi A: Pulmonary mucormycosis with cervical lymph node involvement in a patient with acute myeloid leukaemia: A case report. Mycoses 51: 354-356, 2008

34. Sharma A, Gupta V, Singh RS, Kakkar N, Singh S and Bambery P Angioinvasive pulmonary mucormycosis presenting as multiple bilateral pulmonary nodules in a patient without obvious predisposing factors. Singapore Med J 49: e269-e271, 2008.

35. Schneidawind D, Nann D, Vogel W, Faul C, Fend F, Horger M, Kanz L and Bethge W: Allogeneic hematopoietic cell transplantation in patients with acute myeloid leukemia and pulmonary mucormycosis. Transpl Infect Dis 14: E166-E172, 2012.

36. Fitzpatrick MC and Carter BW: Pulmonary mucormycosis complicating cutaneous blastic plasmacytoid dendritic cell neoplasm. Proc (Bayl Univ Med Cent) 25: 287-288, 2012.
37. Serio B, Rosamilio R, Giudice V, Zeppa P, Esposito S, Fontana R, Annunziata $S$ and Selleri C: Successful management of pulmonary mucormycosis with liposomal amphotericin B and surgery treatment: A case report. Infez Med 20 (Suppl 2): S43-S47, 2012.

38. Björkholm M, Runarsson G, Celsing F, Kalin M, Petrini B and Engervall P: Liposomal amphotericin B and surgery in the successful treatment of invasive pulmonary mucormycosis in a patient with acute T-lymphoblastic leukemia. Scand J Infect Dis 33: 316-319, 2001.

39. Li JY, Yong TY, Jurisevic CA, Russ GR, Grove DI, Coates PT and Disney AP: Successful treatment of pulmonary mucormycosis in a renal transplant recipient with limited pulmonary reserve by combined medical and surgical therapy. Heart Lung Circ 18: 226-228, 2009.

40. Agarwal R, Kumar V and Gupta D: Pulmonary mucormycosis: Two of a kind. Eur J Intern Med 17: 63-65, 2006.

41. Xia ZK, Wang WL and Yang RY: Slowly progressive cutaneous, rhinofacial and pulmonary mucormycosis caused by mucor irregularis in an immunocompetent woman. Clin Infect Dis 56: 993-995, 2013.

42. Chacko B, David VG, Tamilarasi V, Deepti AN and John GT: Pulmonary mucormycosis in a nondiabetic renal allograft recipient successfully managed by medical therapy alone. Transplantation 83: 1656-1657, 2007.

43. Petrikkos G, Skiada A, Lortholary O, Roilides E, Walsh TJ and Kontoyiannis DP: Epidemiology and clinical manifestations of mucormycosis. Clin Infect Dis 54 (Suppl 1): S23-S34, 2012.

44. Chamilos G, Marom EM, Lewis RE, Lionakis MS and Kontoyiannis DP: Predictors of pulmonary zygomycosis versus invasive pulmonary aspergillosis in patients with cancer. Clin Infect Dis 41: 60-66, 2005

45. Yagi S, Miyashita N, Fukuda M, Obase Y, Yoshida K, Miyauchi A, Kawasaki K, Soda H and Oka M: Pulmonary mucormycosis (Cunninghamella bertholletiae) with cavitation diagnosed using ultra-thin fibre-optic bronchoscopy. Respirology 13: 312-314, 2008.

46. Hua-Ping Z, Jian L, Jing-Bin H, Jie G, Guo-Xin M, Yan-Hong J and Li-Xin X: Surgical resection and liposomal amphotericin B to treat cavitary pulmonary zygomycosis in a patient with diabetes. Respir Care 56: 1837-1931, 2011. 